\title{
Unsupervised Learning of Head Pose through Spike-Timing Dependent Plasticity
}

\author{
Ulrich Weidenbacher and Heiko Neumann \\ University of Ulm, Institute of Neural Information Processing, 89069 Ulm \\ \{ulrich. weidenbacher, heiko.neumann\}@uni-ulm.de
}

\begin{abstract}
We present a biologically inspired model for learning prototypical representations of head poses. The model employs populations of integrate-and-fire neurons and operates in the temporal domain. Timesto-first spike (latencies) are used to develop a rank-order code, which is invariant to global contrast and brightness changes. Our model consists of 3 layers. In the first layer, populations of Gabor filters are used to extract feature maps from the input image. Filter activities are converted into spike latencies to determine their temporal spike order. In layer 2, intermediate level neurons respond selectively to feature combinations that are statistically significant in the presented image dataset. Synaptic connectivity between layer 1 and 2 is adapted by a mechanism of spike-timing dependent plasticity (STDP). This mechanism realises an unsupervised Hebbian learning scheme that modifies synaptic weights according to their timing between pre- and postsynaptic spike. The third layer employs a radial basis function (RBF) classifier to evaluate neural responses from layer 2 . Our results show quantitatively that the network performs well in discriminating between 9 different input poses gathered from 200 subjects.
\end{abstract}

\section{Introduction}

\subsection{Motivation}

Over the last decades, several researchers put their effort on the investigation of methods to reliably estimate gaze directions of persons attending to certain objects or persons. This field of research is closely linked to other face related topics such as face detection, face recognition or facial expression analysis since most of them have to account for pose variations (see [Zhao et al., 2000] for a literature survey).

Here, we focus on the reliable estimation of head pose from monocular greyscale images since this question seems to be very crucial for all fields of research that are involved in human-machine-interaction (HMI) [Weidenbacher et al., 2006] or human-robot-interaction (HRI) [Nagai, 2005]. Furthermore, in a car driver scenario, it would be very informative to know which direction the driver is attending to in order generate warnings in case of an unattended threat [Beardsley, 1998]. Interestingly, humans have remarkable skills in making 
reliable pose discriminations [Poppe et al., 2007], as it can be observed in, e.g., conversations to estimate in which direction a dialog partner is attending to [Strauss, 2006]. There are quite a number of existing methods for pose estimation which can be divided into two different categories: Model-based approaches try to find landmarks points in the face to build a pose specific graph [Krüger et al., 1997, Vatahska et al., 2007] . While appearance-based approaches use the whole image of the face to estimate head pose using PCA [Pentland et al., 1994, Cootes et al. 1998] or neural networks [Voit et al., 2007]. The advantage of appearance-based models is that no facial landmarks have to be detected which is often a problem due to occlusion when the face is in profile view or an eye is covered by hairs. On the other hand, appearance-based approaches need more training data to achieve sufficient performance.

\subsection{Biological Plausibility}

In the last ten years, it became more an more clear that the development of new machine learning algorithms alone might not be the best way to solve recognition problems, and that the feature set used has a strong impact on the performance of these appearance based algorithms [Meyers and Wolf, 2008]. Since the human visual system can perform pose discrimination tasks at a level of high accuracy [Poppe et al., 2007], it seems natural to try to understand and emulate how it represents visual data in order to derive features that will be useful in computer vision systems. Recently, [Masquelier and Thorpe, 2007] have proposed a biologically motivated model for learning visual features that was also inspired by the well-known Riesenhuber \& Poggio model [Riesenhuber and Poggio, 1999] in which high level features such as object categories are composed in a hierarchical fashion from low-level features such as simple contrast detectors. While most of the neurally inspired models interpret activities (e.g. filter responses) as spike rates, Thorpe and colleagues use the temporal order of incoming spikes (rank-order) as an efficient code. This offers the advantage to use spike-timebased learning techniques such as STDP, a Hebbian learning rule that changes synaptic weights based on the timing between pre- and postsynaptic spike time [Bi and Poo, 2001]. This kind of coding strategy has been already tested successfully for face identification [Delorme and Thorpe, 2001].

\subsection{Brief Overview}

In this contribution, we present an extended version of the model proposed by [Masquelier and Thorpe, 2007] to learn pose specific neural representations. They incorporate STDP which is known to have the effect of concentrating high synaptic weights on afferents that systematically fire early [Guyonneau et al., 2005]. Moreover, the authors trained their model with images of different categorical objects in front of changing backgrounds. The model finds statistical regularities in the feature domain which are used in combination with a radial basis function classifier (RBF) to discriminate between different object classes (faces, motorbikes, and background). We provided the model with additional 
feature dimensions (scale and phase) to learn pose specific representations of faces. This is a more challenging task as in-class discriminations in general are harder than between-class discriminations. More clearly, a face in frontal pose has more similarity with a slightly rotated face than a face with a motorbike.

\section{Methods}

\subsection{Model Outline}

A greyscale intensity image is filtered with Gabor filters of different orientation, scale and phase polarity, followed by a local normalisation (shunting inhibition). This leads to 48 feature maps providing feature specific neural activity. These activities are further converted into spike ranks by sorting all activities over all feature maps in descending order. In the following layers, only spike ranks, i.e., the temporal order of spiking neurons is used. Spikes are propagated consecutively from layer 1 through STDP synapses to layer 2, where integrate-and-fire neurons compete to elicit their first spike. The prototype who fires first is the winner, whose weights are updated according to the STDP rule (winner-takeall). Final pose classification is done in layer 3 by evaluating a trained RBF classifier using binary pose labels and layer 2 responses as input. The major steps of the model are depicted in Fig. 1.

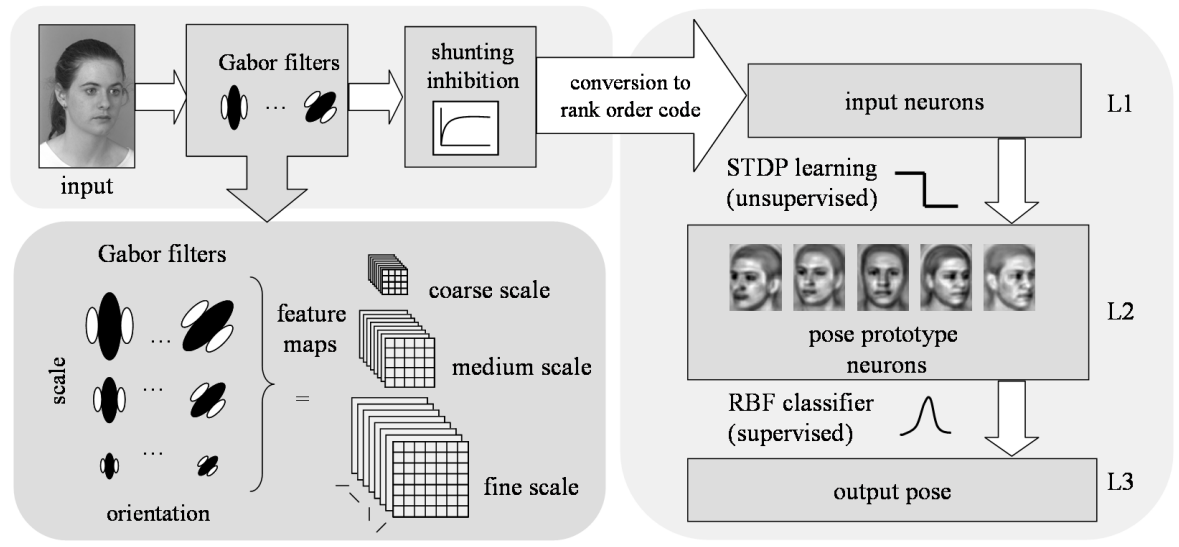

Fig. 1. Overview of the model. Our model consists of three major processing steps. Beginning with a preprocessing stage low level features are extracted and further converted into a rank-order code. Following, pose prototypes are learned from these temporal codes by applying an unsupervised STDP specific leaning rule. After learning has converged, an RBF classifier is used to evaluate the responses from prototypical pose neurons. 


\subsection{Feature Extraction}

The initial stage of processing face images is represented by Gabor wavelets functions [Daugman, 1988] which resembles simple cells in the primary visual cortex. A Gabor filter bank applied to an input image results in different feature maps which selectively respond according to their feature type. Here we used 8 orientations, 3 scales and 2 phases (ON/OFF centre receptive field, responding to positive and negative local contrast) resulting in 48 feature maps (Fig. 1). Furthermore we apply shunting inhibition [Sperling, 1970] to each feature map which has the effect of normalizing filter activity in a spatial neighbourhood within individual feature maps.

\subsection{Rank-Order Code}

Filter responses from each feature map can be interpreted as neural activity. The more strongly a cell is activated the earlier it fires. This conversion from activity to latency is in accordance with physiological data that response latency decreases with increases in stimulus contrast [Albrecht et al., 2002]. [Thorpe, 1990] has proposed to use the temporal order of spike latencies rather than the latencies themselves. This has several advantages: first keeping only the temporal oder saves memory (and computational time) and second the code becomes invariant against global brightness and contrast changes. Such a rank-order code is invariant against global contrast changes since it only represents the order but not the precise times to first spike.

\subsection{Model Dynamics and STDP learning}

Input neurons are connected with each neuron in layer 2 via connections which define a retinotopic map. Synaptic weights between layer 1 and layer 2 neurons are initially set to 0.5 augmented by Gaussian distributed noise $(\sigma=0.01)$. Layer 1 (presynaptic) neurons subsequently fire a single spike in temporal order according to their rank. Layer 2 neurons integrate postsynaptic potentials determined by weights that are connected to the firing presynaptic neuron over time until the postsynaptic neuron itself reaches its firing threshold. As soon as the first postsynaptic neuron fires, a winner-take-all mechanism takes place and prevents all other layer 2 neurons from firing. Weights of the winner neuron are now updated according to the simplified binary STDP rule adapted from [Masquelier and Thorpe, 2007]

$$
\begin{array}{ll}
\Delta w=a^{+} \cdot w \cdot(1-w), \text { if } & t_{i}-t_{j} \leq 0 \\
\Delta w=a^{-} \cdot w \cdot(1-w), \text { if } & t_{i}-t_{j}>0
\end{array}
$$

where $\mathrm{i}$ and $\mathrm{j}$ refer to the pre- and postsynaptic neurons, respectively, $t_{i}$ and $t_{j}$ are the corresponding spike ranks. $\Delta \mathrm{w}$ is the synaptic weight modification and $a^{+}$ and $a^{-}$are the two parameters specifying the amount of change for potentiation and depression of connection weight, respectively. Note, that the learning rule assures that weights remain in range $[0,1]$, therefore no uncontrolled growth of weights can occur. 


\subsection{RBF Classifier}

To evaluate the responses of self-organised prototype neurons, we used a radial basis function classifier. The RBF network was trained with vectors of postsynaptic spike latencies (i.e. the number of presynaptic spikes that were necessary to trigger the spike of a layer 2 neuron). As teacher signal, we used binary class labels from the FERET pose dataset [Phillips et al., 2000]. Training of the RBF classifier did not start until learning of STDP synapses of layer 1-to-layer2 connectivities had finished.

\section{Model Simulations}

As input of the model we used images of 200 subjects in 9 different poses taken from the FERET database [Phillips et al., 2000]. The size of the input images was $72 \times 64$ pixels. In our simulations we used 9 pose neurons initialised with 0.5 in combination with an additive Gaussian noise $\operatorname{term}(\sigma=0.01, \mu=0)$. Receptive field sizes (RF) of layer 2 pose neurons were set to $65 \times 55$ pixels. RFs were duplicated in overlapping retinotopic maps (2 pixels steps), in order to preserve translation invariance. The threshold for the pose neurons to emit a spike was set to $10 \%$, i.e., when the sum of integrated STDP weights exceeded $10 \%$ of the total number of weights within the receptive field ${ }^{1}$. The learning rates $a^{+}$and $a^{-}$in Eq. 1 were set to $2^{-5}$ and $-0.7 \cdot 2^{-5}$. After subsequent presentations of faces in arbitrary poses, neurons in layer 2 began to show a preference for specific head poses (Fig. 2). After 4000 learning steps, weights had converged to a stable state (Fig. 3).

To evaluate the resulting pose prototypes, we turned off learning and again processed all images of the database until the stage of layer 2 where each pose neuron reached a certain potential depending on its pose preference. Note that up to this point, solely unsupervised learning was performed, meaning that no external teacher signal was given to the model. Consequently, the model had no knowledge about which prototype neuron is related to which pose. Poses were only learned due to hidden statistical regularities in the dataset. This vector of postsynaptic potentials was then used as input code to train an RBF classifier. As a teacher signal, we provided the RBF classifier with pose class memberships of each input image. After successful training of the RBF classifier, the output neuron of the RBF that responded maximally determines the pose class of the input image. We performed a 200-fold cross-validation on the dataset to evaluate the performance of the multi-class RBF classifier. More clearly, we tested each of the 9 poses from one subject which the system had never seen before while the system was trained with the remaining 199 subjects (including all poses). Then we compared the pose responses from the RBF network with the correct pose. Fig. 4 illustrates the confusion matrix which demonstrates that the vast majority of the data had been classified correctly. In quantitative terms $94.7 \%$ of all poses were classified within $+/$ - one pose class around the correct one.

\footnotetext{
${ }^{1}$ Exact parameter setting was not critical for the system
} 


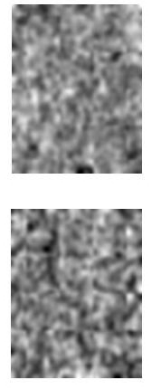

0
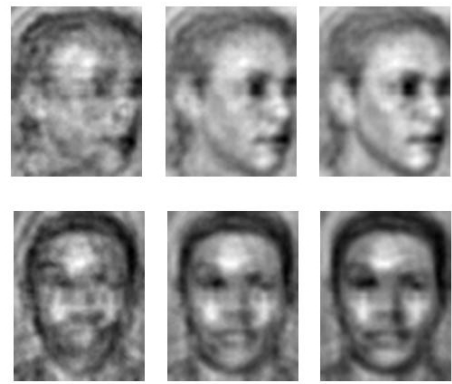

10

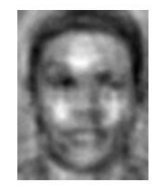

20

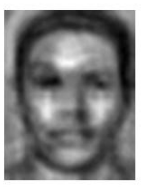

50
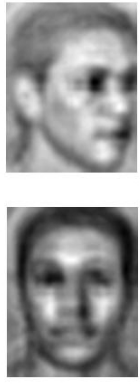

200

number of of learning steps

Fig. 2. The figure shows snapshots over time of reconstructed receptive fields for two example pose neurons. Both neurons become selective for distinct head poses. After 200 postsynaptic spikes (weight changes) the weights remain stable.

a)
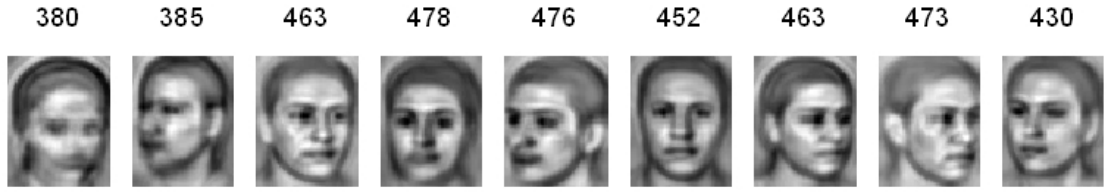

b)
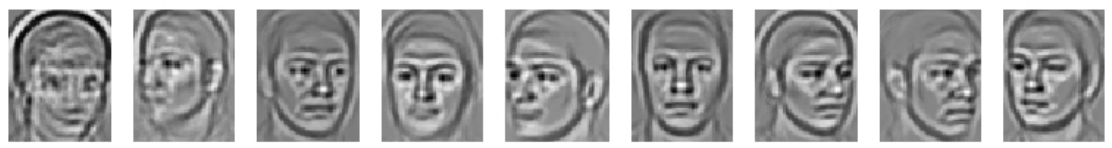

c)
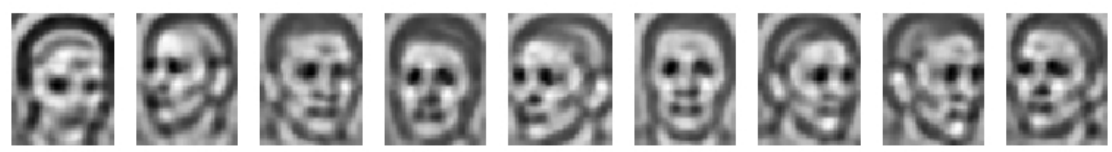

d)
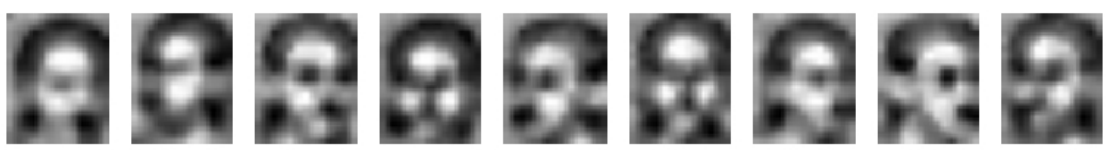

Fig. 3. Receptive fields of all layer 2 neurons after 4000 learning steps. Images in a) show additive reconstructions of learned weights from all feature channels (scale, orientation and phase polarity). b) - d) show weight distribution separate for each Gabor scale from fine to coarse. It is clearly visible that layer 2 neurons show a preference for particular head poses. The numbers over each column denote the quantity of learning steps for each neuron. 


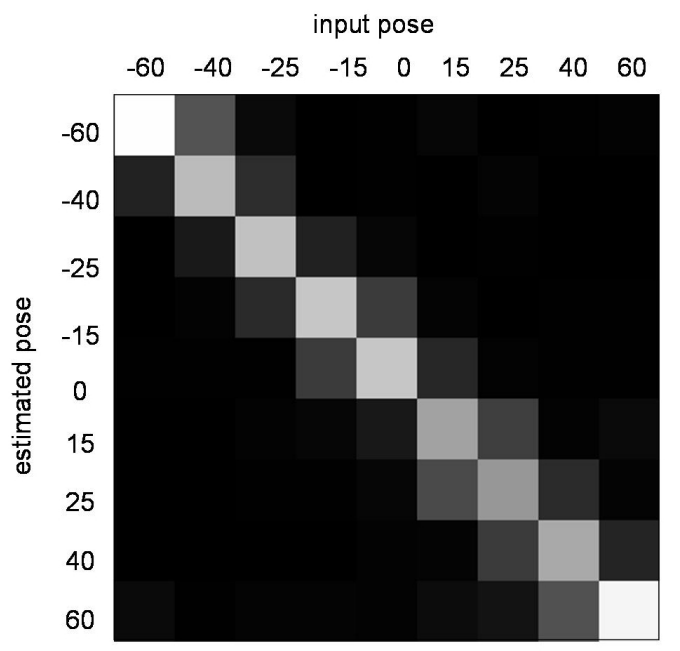

Fig. 4. Confusion matrix of RBF classifier output using a 200-fold cross-validation. The figure shows that nearly all pose classification results lie within the range of $+/$ one pose class around the correct pose (corresponding to $94.7 \%$ classifications).

\section{Discussion \& Conclusion}

We have presented a model that learns pose selective representations from feature ensembles of oriented faces. Moreover we have shown, that this intermediate complexity level representation can be used as input for a final RBF classifier in order to produce consistent pose estimates. This type of hybrid model has several advantages compared to other leaning approaches such as e.g. back propagation networks. First, selectivity in the STDP layer for significant input patterns is achieved after only few learning steps (cp. Fig. 2). Second, there is no problem to deal with local minima and finally, the intermediate level representation of a pose does not depend on an external teacher signal. This means that in principle all significant variations of the dataset are captured by an unsupervised learning process. This is closely linked to principal component analysis (PCA) [Pentland et al., 1994] an alternitive unsupervised learning approach where a system of orthogonal eigenvectors represents significant second-order variations in the data represented by prototypes that are termed eigenfaces. However, the eigenfaces approach is based on the rate code assumption and therefore does not take temporal correlations into account. Moreover, eigenfaces are restricted to be orthogonal to each other.

A problem that many models are confronted with is to find an optimal parameter setting to achieve accurate behaviour. That is in our model, for instance, to choose the number of postsynaptic neurons in layer 2 of the model. It is unclear how many neurons are needed to optimally capture a certain property from the data such as pose. Furthermore, the threshold for a postsynaptic neuron to 
emit a spike has also strong influence on the learning behaviour, since too small thresholds would only focus on the very strongest contrasts (earliest spikes) while high thresholds would cause STDP to loose focus on the most important features and selectivity will get worse.

We plan to further improve the model by developing a scheme for dynamic prototype allocation. This would have the benefit that the user would not have to select a static number of STDP neurons prior to the learning. Moreover, we will investigate the extension of the model to learn combinations of patterns (such as eyes, nose and mouth) and their spatial relationships as it is proposed, e.g., in [Krüger et al., 1997]. Furthermore, we are currently experimenting with video sequences of continuous pose variations which constitute a more natural input pattern than randomly shuffled images. Finally, with these continuous frame sequences optical flow estimates could also be taken into account.

\section{Acknowledgments}

This work was supported by a grant from the Ministry of Science and Arts of Baden-Württemberg, Germany.(Az.: 32-7532.24-14-19/3) to Heiko Neumann.

\section{References}

[Albrecht et al., 2002] Albrecht, D., Geisler, W., Frazor, R., and Crane, A. (2002). Visual cortex neurons of monkey and cats: Temporal dynamics of the contrast response fucntion. Neurophysiology, 88:888-913.

[Beardsley, 1998] Beardsley, P. A. (1998). A qualitative approach to classifying head and eye pose. In IEEE Workshop on Applications of Computer Vision, page 208. IEEE Computer Society.

[Bi and Poo, 2001] Bi, G. and Poo, M. (2001). Synaptic modification by correlated activity: Hebb's postulate revisited. Annu. Revi. Neurosci., 24:139-66.

[Cootes et al., 1998] Cootes, T. F., Edwards, G. J., and Taylor, C. J. (1998). Active appearance models. In Proc. of the European Conference on Computer Vision, volume 2, pages 484-498.

[Daugman, 1988] Daugman, J. (1988). Complete discrete 2d gabor transforms by neural networks for image analysis and compression. Transactions on Acoustics, Speech, and Signal Processing, 36(7):1169-1179.

[Delorme and Thorpe, 2001] Delorme, A. and Thorpe, S. (2001). Face identification using one spike per neuron. Neural Networks, 14:795-803.

[Guyonneau et al., 2005] Guyonneau, R., van Rullen, R., and Thorpe, S. (2005). Neurons tuned to the earliest spikes through stdp. Neural Computation, 17(4):859-879.

[Krüger et al., 1997] Krüger, N., Pötzsch, M., and von der Malsburg, C. (1997). Determination of face position and pose with a learned representation based on labelled graphs. Image Vision Comput., 15(8):665-673.

[Masquelier and Thorpe, 2007] Masquelier, T. and Thorpe, S. (2007). Unsupervised learning of visual features through spike timing dependent plasticity. PLoS Comput Biol 3(2), 3(2):e31.

[Meyers and Wolf, 2008] Meyers, E. and Wolf, L. (2008). Using biologically inspiered features for face processing. International Journal of Computer Vision, 76:93-104. 
[Nagai, 2005] Nagai, Y. (2005). The role of motion information in learning humanrobot joint attention. In Proceedings of the 2005 IEEE International Conference on Robotics and Automation, pages 2069-2074.

[Pentland et al., 1994] Pentland, A., Moghaddam, B., and Starner, T. (1994). Viewbased and modular eigenspaces for face recognition. IEEE Conference on Computer Vision and Pattern Recognition.

[Phillips et al., 2000] Phillips, P., Moon, H., Rauss, P., and Rizvi, S. (2000). The feret evaluation methodology for face recognition algorythems. IEEE Transactions on Pattern Analysis and Machine Intelligence, 22(10):1090-1104.

[Poppe et al., 2007] Poppe, R., Rienks, R., and Heylen, D. (2007). Accuracy of head orientation perception in triadic situations: Experiment in a virtual environment. Perception, 36(7):971-979.

[Riesenhuber and Poggio, 1999] Riesenhuber, M. and Poggio, T. (1999). Hierarchical models of object recognition in cortex. Nature Neuroscience, 2:1019-1025.

[Sperling, 1970] Sperling, G. (1970). Model of visual adaptation and contrast detection. Perception and Psychophysic, 8:143-157.

[Strauss, 2006] Strauss, P.-M. (2006). A slds for perception and interaction in multiuser environments. 2nd Intl Conf. on Intelligent Environments, pages 171-174.

[Thorpe, 1990] Thorpe, S. (1990). Parallel processing in neural systems and computers, chapter Spike arrival times: A highly efficient coding scheme for neural networks, pages 91-94. Elsevier.

[Vatahska et al., 2007] Vatahska, T., Bennewitz, M., and Behnke, S. (2007). Feature based head pose estimation from images. IEEE Conf. on Humanoid Robots.

[Voit et al., 2007] Voit, M., Nickel, K., and Stiefelhagen, R. (2007). Neural networkbased head pose estimation and multi-view fusion. LNCS 4122, pages 291-298.

[Weidenbacher et al., 2006] Weidenbacher, U., Layher, G., Bayerl, P., and Neumann, H. (2006). Detection of head and gaze direction for human-computer interaction. LNCS, 4021:9-19.

[Zhao et al., 2000] Zhao, W., Chellappa, R., Rosenfeld, A., and Phillips, P. (2000). Face recognition: A literature survey. ACM Computing Surveys, 35(4):399-458. 